\title{
10-Year Experience with Reinforced Ureteral Stents for Malignant Ureteral Obstruction
}

\author{
Benoît Vogt (iD) \\ Laure-Hélène Blanchet ${ }^{2}$ \\ 'Department of Urology, Polyclinique de \\ Blois, La Chaussée Saint-Victor, 4I260, \\ France; ${ }^{2}$ Department of Public Health, \\ Hôpital Saint-Antoine (AP-HP), Paris, \\ 750I2, France
}

\begin{abstract}
Introduction: Malignant ureteral obstruction (MUO) hinders the management of malignant diseases. Indwelling stent is a common method to release renal obstruction, but stent failure with obstruction is frequent. The studies conclude that stent obstruction divides survival by 2 or even 4. We carried out the present study in order to evaluate drainage efficiency and overall survival by using stents with distinctive degrees of stiffness.

Materials and Methods: We performed an analysis of 156 patients with MUO drainage at a single institution from June 2009 to June 2019.

Results: Of the 156 patients, $128(82.1 \%)$ died with a mean survival time of $15.3 \pm 14.4$ months after the first ureteral stent procedure (USP). In order to study stent failure and overall survival, the patients were divided into 3 groups. Group 1 with patients died soon after only one USP $(n=37)$. Group 2 with patients had more than one USP and no obstruction $(n=41)$. Patients in Group 3 had more than one USP and at least one stent obstruction $(n=62)$. During the study period, 556 USPs were analysed separately. The stent failure with obstruction occurred in $23.0 \%(128 / 556)$ of USP at a mean of $4.4 \pm 3.6$ months. In case of stent failure, a progressive choice of stents was shaped to overcome each failure by focusing on the criterion of increasingly stiff stents. Patients in Group 1 died soon at mean of $4.9 \pm 4.8$ months. The mean survival time of patients in Groups 2 and 3 were, respectively, $19.4 \pm 11.2$ and $21.5 \pm 16.3$ months $(\mathrm{P}=0.19)$.

Conclusion: MUO is a serious disease but this study is the first to prevent survival rate from falling by choosing the stent stiffness suitable for the patient. The active detection of stent failure has been shown to be essential for preserving survival.
\end{abstract}

Keywords: malignant ureteral obstruction, survival, ureteral stent, tandem ureteral stent, neoplasms, renal failure

\section{Introduction}

Malignant ureteral obstruction (MUO) caused by malignant diseases may require an indwelling stent. The mean time from cancer diagnosis to first stent placement has been reported to be approximately 34 months. ${ }^{1}$ Ureteral obstruction may induce renal failure, renal colic or pyelonephritis. Chronic renal insufficiency is a barrier to several therapies, including chemotherapy. Indwelling stent is the common method to release renal obstructions, but adequate stent insertion across an obstructed ureter does not necessarily guarantee renal decompression. ${ }^{2-4}$ Most studies report an approximately $28 \%$ failure rate, which hampers the management of malignant diseases and the need for repeated stent changes may cause a significant reduction in overall quality of life., ${ }^{2,5}$ Moreover, most studies conclude that stent obstruction divides survival by 2 or even 4 or at least deteriorates renal function over time, which may lead to dialysis. ${ }^{1,6-9}$
Correspondence: Benoît Vogt Department of Urology, Polyclinique de Blois, I rue Robert Debré, La Chaussée Saint-Victor, 41260, France

Tel +332549065 II

Fax +33254906566

Email message@benoitvogt.fr 
We carried out the present study in order to evaluate drainage efficiency and overall survival by using stents with distinctive degrees of stiffness.

\section{Materials and Methods}

From June 2009 to June 2019 in a single institution, a total of 156 consecutive patients were treated for the discovery of a MUO. One hundred and fifty patients requiring indwelling stent for MUO were fitted with commercially available reinforced ureteral stents. Due to the repetition of high rate of stent failures with obstruction in patients with MUO, the progressive and prospective choices of stents was shaped by a single surgeon (BV) to overcome each failure by focusing on the criterion of increasingly stiff stents.

The causes of the extrinsic malignant ureteral compression were determined by history and radiographic data. Ureteral obstruction was defined by the presence of renal colic or renal failure with increased serum creatinine or hydronephrosis confirmed by computerized tomography or ultrasound with/without pyelonephritis.

The operation was performed under general or regional anesthesia and was performed mostly by the same surgeon. A bladder resection was performed to discover the ureteral orifice if needed. The ureteral stent was placed in the kidney under direct vision through the cystoscope and fluoroscopic guidance. The wire guide was a stiff or an extra stiff guide (Lunderquist Extra Stiff Wire Guide, $0.89 \mathrm{~mm}$, Cook Medical). In case of tandem insertion, the tandem stents were pushed simultaneously into the ureter. No balloon dilation was used. When cystoscopy with insertion of a retrograde stent failed, the cystoscopy coupled with percutaneous nephrostomy (PCN) allowed antegrade stenting.

The efficiency of drainage was confirmed postoperatively by regression of pain or renal failure with decreased serum creatinine, and improvement in the degree of hydronephrosis.

The patient received a document advising her/him to perform a renal ultrasound during the 5th and 6th month for routine oncologic surveillance and to change the stent every 6 months.

Stent failure was defined as the presence of renal colic or renal failure with increased serum creatinine or worsening hydronephrosis during routine oncologic surveillance with/without pyelonephritis.

A successful stent insertion attempt contributed to one ureteral stent procedure (USP). Patients with bilateral stents contributed only one observation to the dataset. Bilateral stents in the same patient with unilateral or bilateral stent failure were counted as a single stent failure. There was no selection or exclusion, and all patients were included, even those with poor performance status.

Double-pigtail stents $8 \mathrm{~F}$ without holes, such as Coloplast Vortek Tumor Stents, Bard Angiomed Urosoft Tumor Stents, Teleflex Medical Tumor Stents, and Coloplast Neoplex ureteral stents were used in our study. The progressive choice of stents was shaped to overcome each failure by focusing on the criterion of increasingly stiff stents. As the previous study confirmed, the radial compressive stress of the Teleflex stents $\left(5.4 \mathrm{~N} . \mathrm{mm}^{-2}\right)$ was higher than with the Bard stents (around $2.8 \mathrm{~N} . \mathrm{mm}^{-2}$ ) and the Coloplast stents $\left(1.4 \mathrm{~N} . \mathrm{mm}^{-2}\right) .{ }^{10}$ Obstruction of the stiffest stent in less than 6 months motivated the switch to $8 \mathrm{~F}$ tandem stents.

The data are presented as mean \pm SD. Data were analysed using Student t, Fisher exact and Pearson's Chisquared. Kaplan-Meier survival curves were generated to estimate overall survival and were compared by the Log rank test. Statistical analyses were performed using software R. Values of $\mathrm{P}<0.05$ were considered significant.

\section{Results}

The study population consisted of 156 patients (96 men and 60 women) with a mean age of $74.6 \pm 12.6$ years. The malignant disease was progressive in $90.4 \%(141 / 156)$ of patients. The obstruction was bilateral in $57.7 \%$ of cases. One hundred and twenty-eight patients $(82.1 \%)$ had previous chemotherapy or hormonal therapy and 71 patients (45.5\%) had previous radiotherapy.

Of the 156 patients, $128(82.1 \%)$ died with a mean survival time of $15.3 \pm 14.4$ months after the first USP, and $71(55.5 \%)$ died within a year with a median of 10.5 months.

At the endpoint of our study, 28 patients (17.9\%) are alive with a mean follow-up (range) of $48.8 \pm 29.6$ months (1-10 years).

The most common causes of malignancy were prostate cancer $(28.6 \%)$, followed by bladder cancer $(19.3 \%)$ and cervical cancer (14.3\%) (Table 1).

Of the 156 patients, 150 were fitted with ureteral stents, 4 by $\mathrm{PCN}$ alone, and 2 were not treated for poor performance status. The patients drained by PCN alone had advanced metastatic cancer and died at a mean of $2.7 \pm$ 1.2 months after the diagnosis of MUO. The other two patients died in less than a month. 
Table I Distribution of the Type of Malignancy of the Three Groups

\begin{tabular}{|c|c|c|c|c|}
\hline Type of Malignancy & Total n (\%) & Group I $(n=37)$ & Group $2(n=4 I)$ & Group $3(n=62)$ \\
\hline Prostate cancer & $40(28.6)$ & 10 & 15 & 15 \\
\hline Bladder cancer & $27(19.3)$ & 7 & 14 & $6 *$ \\
\hline Cervical cancer & $20(14.3)$ & 2 & 1 & $17 * *$ \\
\hline Colonic cancer & $16(11.4)$ & 6 & 3 & 7 \\
\hline Rectal cancer & II (7.9) & 5 & 2 & 4 \\
\hline Ovary cancer & $8(5.7)$ & 2 & 2 & 4 \\
\hline Pelvic cancer & $5(3.6)$ & 0 & I & 4 \\
\hline Breast cancer & $5(3.6)$ & 2 & 1 & 2 \\
\hline Retroperitoneal mass & $4(2.8)$ & 1 & 1 & 2 \\
\hline Gastric cancer & $2(1.4)$ & I & I & 0 \\
\hline Oesophageal cancer & $\mathrm{I}(0.7)$ & 0 & 0 & I \\
\hline Lung cancer & I (0.7) & I & 0 & 0 \\
\hline
\end{tabular}

Notes: $* \mathrm{P}<0.002$ versus group 2 and $* * \mathrm{P}<0.001$ versus group 2 .

Of the 150 patients fitted with ureteral stents, the retrograde stenting by endoscopy was unsuccessful in 15 patients $(10.0 \%)$ and the stents were inserted by a coupled endoscopic and PCN route. The design of the stent was double-pigtail stents in 99 patients $(63.5 \%)$ and $\mathrm{JFil}^{\circledR}$ or customized ureteral stent in 36 patients $(36.5 \%) .{ }^{11,12}$

During the study period, 12 patients subsequently underwent ileal conduit urinary diversion (ICUD) and 2, nephrectomy. Among the patients with ICUD, 3 were suggested for recurrent ureteral stent obstruction after radiotherapy for cancer at a mean of $32.7 \pm 16.8$ months after the discovery of MUO. Two are alive and one died of cancer 1 year after the bypass. Nine ICUDs were performed after cystectomy for bladder cancer with MUO at a mean of $2.3 \pm 2.1$ months after diagnosis. Six out of 9 had neoadjuvant chemotherapy and all but one patient died at a mean age of $20.0 \pm 18.0$ months. Of the patients who chose nephrectomy, both patients had cervical cancer and only one was alive.

In order to study stent failure and overall survival, the patients were divided into 3 groups. Group 1 with patients died soon after only one USP $(\mathrm{n}=37)$. Group 2 with patients had more than one USP and no obstruction ( $\mathrm{n}=$ 41). Patients in Group 3 had more than one USP and at least one stent obstruction $(n=62)$. Figure 1 shows the flow diagram with procedures and patient's state.

The 37 patients in Group 1 died at a mean of $4.9 \pm 4.8$ months. The patients had a mean age of $78.3 \pm 11.2$ years, which was significantly older than the other patients of Group 2 and $3(\mathrm{P}<0.01)$. Only 1 patient had a possibility of stent failure leading to death.
Among the 103 patients in Groups 2 and 3 who had a stent change therapy, $62(60.2 \%)$ experienced at least one stent failure. The mean age between Groups 2 and 3 was comparable.

Among the 41 patients in Group 2 without stent failure, four $(9.8 \%)$ were in remission. At 10 years of follow-up, 33 patients $(80.5 \%)$ died with a mean survival time of 19.4 \pm 11.2 months after discovery of MUO. Patients in Group 2 had bladder cancer (34.1 vs 9.7\%, $\mathrm{P}<0.002)$ more often than those in Group 3. The patients required 153 USPs at a mean of $3.7 \pm 2.0$ times per patient with a mean time of stenting of $7.3 \pm 3.2$ months.

Among the 62 patients in Group 3 with stent failure, ten (16.1\%) were in remission. At 10 years of follow-up, 43 patients $(69.4 \%)$ died with a mean survival time of $21.5 \pm$ 16.3 months after discovery of MUO. Patients in Group 3 were more often women (56.4 vs $24.4, \mathrm{P}<0.003$ ) with cervical cancer (27.4 vs $2.4, \mathrm{P}<0.001)$ and radiotherapy (64.5 vs $39.0 \%, \mathrm{P}<0.01$ ) than those in Group 2 . The patients required 356 USPs at a mean of $5.7 \pm 3.9$ times per patient with a mean time of stenting of $5.6 \pm 3.3$ months. The number of USP was significantly higher than in Group $2(\mathrm{P}<0.002)$. Among the 19 patients alive, half were fitted with a tandem stent.

Serum creatinine was collected from patients of Group 3 who had a single episode of stent failure. Among the 31 values, 11 were missing. At the time of procedure for stent failure, the mean serum creatinine was $3.3 \pm 2.5 \mathrm{mg} \mathrm{dl}^{-1}$. After stent change, renal function showed an improvement with a mean serum creatinine at $1.8 \pm 0.9 \mathrm{mg} . \mathrm{dl}^{-1}(\mathrm{P}<0.009)$.

During the study period, 556 USPs were analysed separately. The mean time of stenting was $6.0 \pm 3.4$ 


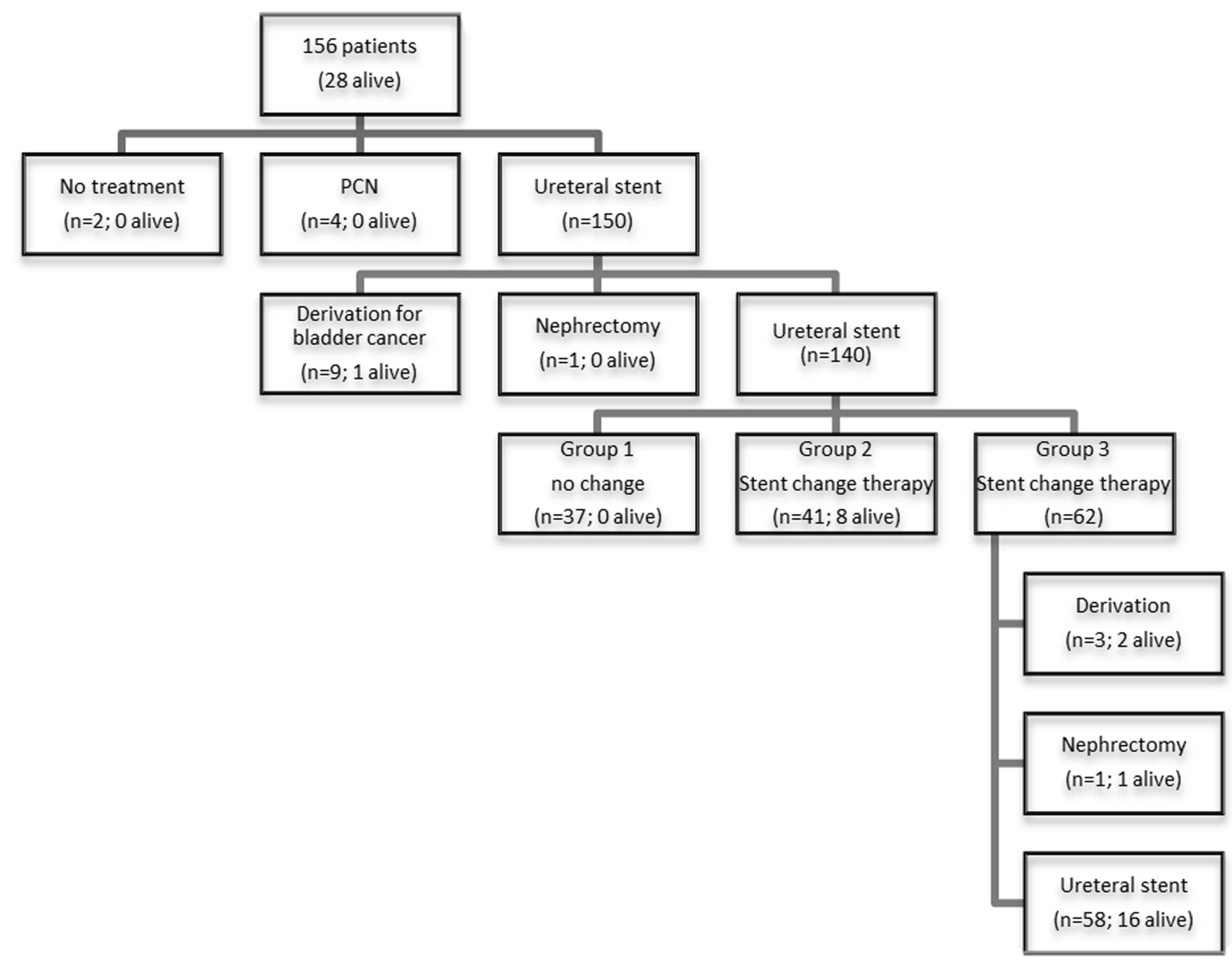

Figure I Flow diagram with procedures and patient's state.

months. The stent failure with obstruction occurred in $23.0 \%(128 / 556)$ of USP at a mean of $4.4 \pm 3.6$ months. Among the patients in Group 3 fitted with the least stiff stents (Coloplast or Bard stents), 53/62 (85.5\%) had at least one stent obstruction. Among the patients in Group 3 fitted with the stiffest ureteral stents (Teleflex stents), 28/ $40(70.0 \%)$ had at least one stent obstruction, while the obstruction occurred in only 2 of 18 patients (11.1\%) with tandem stents $\left(p<4 \cdot 10^{-5}\right)$. The characteristics of the 150 patients of the three groups are described in Table 2.

Kaplan-Meier analysis showed that the difference in the survival between Group 1 and both other groups was statistically significant (Log rank $\mathrm{P}<0.0001$ ). KaplanMeier analysis of Groups 2 and 3 showed that patients with or without stent failure had similar overall survival during follow-up (Log rank $\mathrm{P}=0.19$ ) (Figure 2).

\section{Discussion}

The MUOs are serious events in the development of cancer and hinder the management of malignant diseases. ${ }^{2-4}$ The patients and the causes of malignancy are heterogeneous according to the studies, but the majority of studies observed that mortality is high from $76.2 \%$ to $86.3 \%$ over

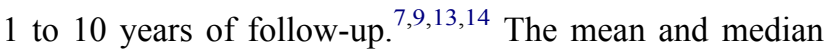
survival were short from 11.1 to 16 months and 4 to 10.7 months, respectively. ${ }^{1,6,7,14-17}$ The results of our study were in agreement with these data.

In our study, ureteral orifice invasion by a bladder cancer appeared to be associated with poor prognosis, and neoadjuvant chemotherapy did not prevent the high $90 \%$ mortality. In addition, the survival of patients with bladder cancer and MUO was 20 months, like that of patients combining other cancers, MUO and stent change 
Table 2 Characteristics of the 150 Patients of the Three Groups. Ten Procedures Followed by lleal Conduit Urinary Diversion Were Excluded

\begin{tabular}{|c|c|c|c|c|}
\hline \multicolumn{5}{|c|}{ Number of Patients (\%) } \\
\hline & Group I $(n=37)$ & Group $2(n=4 I)$ & Group $3(n=62)$ & $P$ value \\
\hline Mean age (years) & $78.3 \pm 11.2$ & $75.4 \pm 11.7$ & $71.4 \pm 13.5$ & $0.01 *$ \\
\hline Gender (men/women) Side & $27 / 10$ & $31 / 10$ & $27 / 35$ & $0.003^{* *}$ \\
\hline Right & 10 & 9 & 9 & \\
\hline Left & 8 & 13 & 14 & \\
\hline Bilateral & 19 & 19 & 39 & \\
\hline Disease progression (\%) & $37(100)$ & $37(90.2)$ & $51(82.3)$ & \\
\hline Previous chemotherapy or hormonal therapy (\%) & $32(86.5)$ & $32(78.0)$ & $55(88.7)$ & \\
\hline Previous radiotherapy (\%) & II (29.7) & $16(39.0)$ & $40(64.5)$ & $0.01 * *$ \\
\hline Urinary derivation (\%) & 0 & $4(6.5)$ & 0 & \\
\hline Survival as endpoint (\%) & 0 & $8(19.5)$ & $19(30.6)$ & \\
\hline Survival time after stenting (months) & $4.9 \pm 4.8$ & $19.4 \pm 11.2$ & $21.5 \pm 16.3$ & $0.2^{* *}$ \\
\hline \multicolumn{5}{|l|}{ Serum creatinine $\left(\mathrm{mg} / \mathrm{dl}^{-1}\right)$} \\
\hline At stenting & & & $3.3 \pm 2.5$ & \\
\hline After stenting & & & $1.8 \pm 0.9$ & 0.009 \\
\hline Number of stent procedures & 37 & 153 & 356 & \\
\hline \multicolumn{5}{|l|}{ Mean number of stent } \\
\hline Procedures per patient & 1.0 & $3.7 \pm 2.0$ & $5.7 \pm 3.9$ & 0.002 \\
\hline Overall stent failure (\%) & 0 & 0 & $128(23.0)$ & \\
\hline
\end{tabular}

Notes: *Group I versus group 2 and $3 .{ }^{* *}$ Group 2 versus group 3.

therapy. Goldfarb et al already observed that for cervical cancer, MUO was associated with a median survival of only 3-6 months and death as endpoint was significantly more common in the stent with cancer patients compared to the non-stent with cancer group. ${ }^{13}$ Ureteral invasion of bladder cancer can reflect an advanced disease, which should be considered serious.

Radiotherapy was observed with significantly higher rates in Group 3 patients with stent failure than in Group 2 patients. Radiotherapy is known for its toxicity on the ureters. In dogs, Van Kampen et al observed that damage to the ureters and the risk of hydronephrosis with ureteral obstruction increased with the dose of radiation. ${ }^{18}$ Other authors reported higher rates of obstruction and stent change of polymeric or metallic stent after radiotherapy. ${ }^{13,19}$

To release the kidney obstruction, $\mathrm{PCN}$ is a procedure in which a drainage tube can be placed under sedation, but dislodgement of the tube with recurrent obstruction is common. ${ }^{20}$ To release the kidney obstruction in case of MUO, most authors favored retrograde stenting. ${ }^{16,17}$ Retrograde stenting failure was consistently described in studies at an average rate of $12.7 \%$ and depended on the severity of tumor invasion of the bladder orifice. In case of stent failure, the most common derivation offered to the patient was PCN. ${ }^{1,15,16,19,21}$ Among the 150 patients in our study, the retrograde stenting by endoscopy was unsuccessful in 15 patients (10.0\%) and the rate of failure was in agreement with these data. However, performing endoscopy with simultaneous PCN allowed anterograde stenting in all cases of the retrograde stenting failure.

Most studies reported stent failure with a mean of $28.7 \%$ at a mean time of 3.0 months. ${ }^{1,5,7,15,16}$ The results at the start of our study were in agreement with these data. However, Figure 2 shows that stent failures decreased at the end of our study by a particular selection of stiff reinforced stents and active screening for insidious stent obstruction.

These obstructions are serious events because over time, they promote renal failure and can even cause death. Indeed, in patients with obstructed ureter after radical cystectomy for bladder cancer, Tombul et al observed that even with active 


\section{Overall survival}

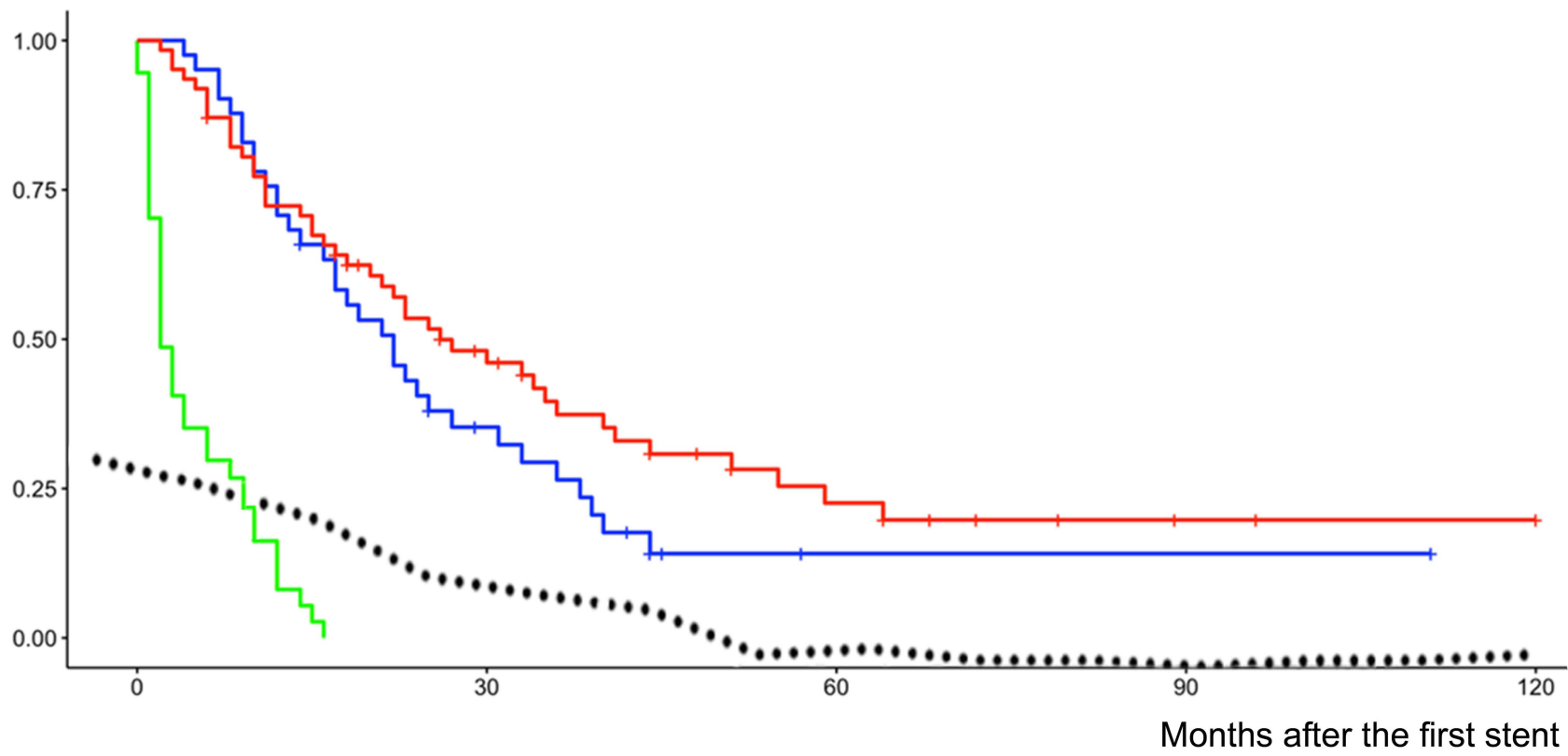

Figure 2 Kaplan-Meier analysis of overall survival after stenting in group I (green curve), group 2 (blue curve) and group 3 (red curve). The dotted curve indicates the decrease in the incidence of stent failure over time. The median time to the occurrence of an event (death or censored) was 2 months with [IC $952-6]$ for group I, 22 months with [IC $\mathrm{IC}_{95}$ 17-33] for group 2, and 26 months with [IC $\left.\mathrm{C}_{95} 20-4 I\right]$ for group 3.

screening for insidious stent obstruction and regular stent changes, kidney function deteriorated over time compared to those who did not develop an obstruction. In addition, deterioration in renal function was even worse in patients with preoperative hydronephrosis. ${ }^{9}$ Elsamra et al showed that patients who experienced stent failure had a mean survival time of 5.8 months, while the unobstructed patients had a mean of 21.3 months. ${ }^{6}$ In another study, even with tandem stents, failure reduced survival time from 5.4 to 1.5 months. ${ }^{8}$ In addition, stent failure may worsen renal failure and Yoon et al reported higher mortality in renal failure. ${ }^{7}$ Finally, stent failure may induce infection and Rosenberg et al reported survival time at 15.3 months and only 11.9 months in case of infection, and if renal failure was not controlled, the median death dropped at 37 days. ${ }^{1}$ Several authors insisted that urologists should be actively involved in the management of patients with MUO to manage stent-related symptoms and monitor for stent failure or decline in renal function. .,13 $^{\text {. }}$

Obstruction of the stiffest stent in less than 6 months motivated the switch to $8 \mathrm{~F}$ tandem stents. Tandem stents have been developed as an alternative to single ureteral stents and the use of tandem stents allowed releasing the renal obstruction in the case of failure of a single stent, testifying that the very design of the stent is clearly involved in the success of drainage., ${ }^{2,6}$ The technique of tandem stent was introduced by Liu et al in 1998. For the authors, the combination of two ureteral stents increases the stiffness and reduces the likelihood of kinking from extrinsic forces. ${ }^{22}$ However, placing two ureteral stents can be a frustrating experience, as one stent may push the other during insertion. ${ }^{23}$ In our study, the tandem stents were pushed simultaneously with extra stiff guides. Among the tandem attempts, only one was unsuccessful. In other studies, tandem attempts failure was $5 \%$ to $27.2 \% .^{14,17,21}$ Several studies showed the benefits of tandem stents but the stents used were variable, and the stents were not reinforced. The permeability was more than $80 \%$ at 3 months and $50 \%$ to $100 \%$ at 6 months. ${ }^{8,14,21,23}$ In our study, stent failure occurred in only $11.1 \%$ of patients fitted with tandem stents and the results seemed better than in the other series, but our tandem stents were $8 \mathrm{~F}$ reinforced stents. Several authors observed a decrease in overall survival in case of tandem failure. ${ }^{6,8}$ Half of the patients alive in Group 3 were fitted with a tandem stent. Even with tandem stents, active clinical and ultrasound screening is crucial to preserve kidney function and patient survival. ${ }^{7,13}$ Patients who still have stent failure even with a tandem stent could require a personalized attitude with a larger and stiffer tube and a higher change frequency. By using stents with distinctive degrees of stiffness, patients with or without stent failure had similar overall survival during follow-up. This study is the first to avoid losing survival by choosing the stent stiffness suitable for the patient. 
Stent-related symptoms have been widely described even in MUO. ${ }^{13,17,20,23}$ In patients with MUO, bladder symptoms may not only be related to the stent itself but also to the preexisting bladder disease. Indeed, Goldfarb et al observed that symptoms in patients with stent and cervical cancer were higher compared to other patients with stent without cancer. ${ }^{13}$ For Rotariu et al, there has been no increase in stent-related symptoms with tandem compared with those produced by single stent. ${ }^{23}$ It has been suggested that changes in the shape of stents could decrease discomfort. ${ }^{11,12}$ Many stent designs, such as biodegradable ${ }^{24}$ or suture stents, ${ }^{11}$ are described or commercialized, but it is essential that the stenosis be drained with a reinforced tube segment for malignant diseases. In previous studies, the innovative pigtail-suture stent (ie, JFil ${ }^{\circledR}$ ) or a customized ureteral stent with a non-refluxing silicone end-piece significantly decreased stent-related symptoms. ${ }^{11,12}$ With the $\mathrm{JFil}^{\circledR}$, no inflammation was seen on the ureter wall around the suture and on the bladder orifice in endoscopy or histological analysis. ${ }^{11,25,26}$ In recent studies including randomization and patient blinding, the results confirmed that suture stent like $\mathrm{JFil}^{\circledR}$ significantly improved patient comfort. ${ }^{27,28}$ About one third of the patients in our study were fitted with these innovative stents. The top priority for future stents suitable for malignant diseases should be to integrate the importance of stent stiffness and shape according to the data of our current knowledge.

\section{Limitations}

\section{Our Study Has Several Limitations}

First, the heterogeneity of patients who were subjected to different treatments according to the severity of the disease and the heterogeneity of MUO with cases with more extensive or severe ureteral obstruction may be subject to selection bias.

Second, our study was neither prospective nor randomized, but its strategy and its results have evolved over time thanks to a progressive and prospective choice of stents, which was shaped by a single surgeon in most procedures. The large number of stent procedures and our 10-year-long follow-up have made it possible to obtain powerful statistical results.

Finally, the choice of the stents was driven by the increase in stiffness, but this criterion was not necessarily the factor actually involved in stent failure and MUO.

The mechanisms leading to stent obstruction remain unknown in this study. It would be useful to know the constriction degree of ureteral stenosis by determining whether urine flow can take place through the extraluminal space between the stent and the ureter wall, or only through the stent lumen. Among the reinforced stents selected in the study, the inner diameter of the stent increased with the stiffness of the stent. ${ }^{10}$ Thus, the stent lumen and its preservation may also be essential parameters to preserve the flow rate. The stiffness would be the only means of keeping the lumen intact. Indeed, by using a mechanical ureteral model, Shilo et al observed that the lumen of the ureteral stent $8 \mathrm{~F}$ offered better patency to colloid solutions than with tandem $7 \mathrm{~F}$ or metal stents. ${ }^{29}$ The debris accumulation is a possible risk factor for stent encrustation, suggesting that larger lumen stents are less likely to become occluded with debris. ${ }^{5,29}$ A study more focused on the specific characteristics of the stents could clarify the impact of the stent stiffness or the stent lumen.

\section{Conclusion}

MUO is a serious disease, but this study is the first to avoid losing survival by choosing the stent stiffness suitable for the patient. The early and active detection of stent failure and the use of stiff stents have been shown to be essential for preserving kidney function and survival. It would be interesting to assess the impact of stent stiffness and stent lumen on overall survival in patients with MUO in a prospective randomized controlled trial.

\section{Abbreviations}

MUO, malignant ureteral obstruction; USP, ureteral stent procedure; ICUD, ileal conduit urinary diversion.

\section{Ethics Approval and Informed Consent}

All procedures performed in studies involving human participants were in accordance with the ethical standards of the institutional and/or national research committee (French Ethical Committee: CPP 17-VOGT-01 and National Medicine Safety Agency: 2017-A00205-48 and IRB registration: 00001072) and with the 1964 Helsinki declaration and its later amendments or comparable ethical standards. Informed consent: Written informed consent was obtained from the patients included in the study.

\section{Acknowledgments}

We thank Professor Janine Dove-Rumé, English Department at the University of Tours, for re-reading the text. 


\section{Author Contributions}

All authors contributed to data analysis, drafting or revising the article, have agreed on the journal to which the article will be submitted, gave final approval for the version to be published, and agreed to be accountable for all aspects of the work.

\section{Disclosure}

The authors certify that all conflicts of interest, including specific financial interests and relationships and affiliations relevant to the subject matter or materials discussed in the manuscript are the following: Benoit Vogt received royalties from Rocamed for the treatment of ureteral stones but there are no financial competing interests in the manuscript. The authors report no other conflicts of interest in this work.

\section{References}

1. Rosenberg BH, Bianco FJ Jr, Wood DP Jr, Triest JA. Stent-change therapy in advanced malignancies with ureteral obstruction. J Endourol. 2005;19(1):63-67. doi:10.1089/end.2005.19.63

2. Elsamra SE, Leavitt DA, Motato HA, et al. Stenting for malignant ureteral obstruction: tandem, metal or metal-mesh stents. Int J Urol. 2015;22(7):629-636. doi:10.1111/iju.12795

3. Vogt B, Desfemmes FN, Desgrippes A. Improving comfort of patients with ureteral obstruction and malignant disease should be of concern. J Palliat Med. 2016;19(11):1132-1133. doi:10.1089/jpm.2016.02763

4. Vogt B. Ureteral stent obstruction and stent's discomfort are not irreparable damages. Urol Case Rep. 2018;29(20):100-101. doi:10.1016/j.eucr.2018.07.025

5. Docimo SG, Dewolf WC. High failure rate of indwelling ureteral stents in patients with extrinsic obstruction: experience at 2 institutions. J Urol. 1989;142(2 Pt 1):277-279. doi:10.1016/s00225347(17)38729-3

6. Elsamra SE, Motato H, Moreira DM, et al. Tandem ureteral stents for the decompression of malignant and benign obstructive uropathy. J Endourol. 2013;27(10):1297-1302. doi:10.1089/end.2013.0281

7. Yoon JH, Park S, Park S, Moon KH, Cheon SH, Kwon T. Renal function is associated with prognosis in stent-change therapy for malignant ureteral obstruction. Investig Clin Urol. 2018;59 (6):376-382. doi:10.4111/icu.2018.59.6.376

8. Varnavas M, Bolgeri M, Mukhtar S, Anson K. The role of tandem double-J ureteral stents in the management of malignant ureteral obstruction. $J$ Endourol. 2016;30(4):465-468. doi:10.1089/ end.2015.0670

9. Tombul ŞT, Sönmez G, Demirtaş A, Tatlışen A. Oncologic and renal function outcomes after radical cystectomy and ureterocutaneostomy: a single center experience. J Urol Surg. 2019;6(4):314-319. doi:10.4274/jus.galenos.2019.2682

10. Vogt B. Stiffness analysis of reinforced ureteral stents against radial compression: in vitro Study. Res Rep Urol. 2020;12:583-591. doi:10.2147/RRU.S285031

11. Vogt B, Desgrippes A, Desfemmes FN. Changing the double-pigtail stent by a new suture stent to improve patient's quality of life: a prospective study. World $J$ Urol. 2015;33:1061-1068. doi:10.1007/s00345-014-1394-2
12. Vogt B. A new customized ureteral stent with nonrefluxing silicone end-piece to alleviate stent-related symptoms in malignant diseases. Urology. 2020;137:45-49. doi:10.1016/j.urology.2019.12.022

13. Goldfarb RA, Fan Y, Jarosek S, Elliott SP. The burden of chronic ureteral stenting in cervical cancer survivors. Int Braz J Urol. 2017;43(1):104-411. doi:10.1590/S1677-5538.IBJU.2016.0667

14. Ozyer U, Dirim A. Tandem ureteral stents in the management of double-J stent dysfunction in gynecological malignancies. Diagn Interv Imaging. 2017;98(9):601-608. doi:10.1016/j.diii.2017.07.005

15. Rosevear HM, Kim SP, Wenzler DL, Faerber GJ, Roberts WW, Wolf JS Jr. Retrograde ureteral stents for extrinsic ureteral obstruction: nine years' experience at University of Michigan. Urology. 2007;70(5):846-850. doi:10.1016/j.urology.2007.07.008

16. Ganatra AM, Loughlin KR. The management of malignant ureteral obstruction treated with ureteral stents. $J$ Urol. 2005;174 (6):2125-2128. doi:10.1097/01.ju.0000181807.56114.b7

17. Haifler M, Shvero A, Zilberman D, et al. Tandem ureteral stents for malignant ureteral obstruction. $J$ Endourol. 2020;34(2):222-226. doi:10.1089/end.2019.0685

18. van Kampen M, Eble MJ, Krempien R, et al. Influence of irradiated volume on ureteral injury after intraoperative radiation therapy: experimental study in dogs. Radiology. 2003;228(1):139-143. doi:10.1148/radiol.2281011963

19. Wang HJ, Lee TY, Luo HL, et al. Application of resonance metallic stents for ureteral obstruction. BJU Int. 2011;108(3):428-432. doi:10.1111/j.1464-410X.2010.09842.x

20. Monsky WL, Molloy C, Jin B, et al. Quality-of-life assessment after palliative interventions to manage malignant ureteral obstruction. Cardiovasc Intervent Radiol. 2013;36(5):1355-1363. doi:10.1007/ s00270-013-0571-9

21. Liu KL, Lee BC, Ye JD, et al. Comparison of single and tandem ureteral stenting for malignant ureteral obstruction: a prospective study of 104 patients. Eur Radiol. 2019;29(2):628-635. doi:10.1007/s00330-018-5560-6

22. Liu JS, Hrebinko RL. The use of 2 ipsilateral ureteral stents for relief of ureteral obstruction from extrinsic compression. J Urol. 1998;159 (1):179-181. doi:10.1016/s0022-5347(01)64050-3

23. Rotariu P, Yohannes P, Alexianu M, et al. Management of malignant extrinsic compression of the ureter by simultaneous placement of two ipsilateral ureteral stents. $J$ Endourol. 2001;15(10):979-983. doi:10.1089/089277901317203047

24. Soria F, de La Cruz JE, Budia A, et al. Iatrogenic ureteral injury treatment with biodegradable antireflux heparin-coated ureteral stent-animal Model Comparative Study. J Endourol. 2021. doi:10.1089/end.2020.0591

25. Vogt B, Chokri I. Histological inflammation in human ureter either healthy or fitted with double-pigtail stent or a thin $0.3 \mathrm{~F}$ suture thread: a Preliminary Study. Adv Urol. 2020;2020:1204897. doi:10.1155/ 2020/1204897

26. Majdalany SE, Aldoukhi AH, Jung H, Mehra R, Roberts WW, Ghani KR. In vivo evaluation of a novel pigtail suture stent. Urology. 2021;148:83-87. doi:10.1016/j.urology.2020.11.031

27. Betschart P, Piller A, Zumstein V, et al. Reduction of stent-associated morbidity by minimizing stent material: a prospective, randomized, single-blind superiority trial assessing a customized 'suture stent'. BJU Int. 2021;127(5):596-605. doi:10.1111/bju.15290

28. Bosio A, Alessandria E, Agosti SC, et al. Pigtail suture stents significantly reduce stent-related symptoms compared to conventional double J Stents: a prospective randomized trial. Eur Urol Open Sci. 2021;29:1-9. doi:10.1016/j.euros.2021.03.011

29. Shilo Y, Modai J, Leibovici D, Dror I, Berkowitz B. Comparative study of renal drainage with different ureteral stents subject to extrinsic ureteral obstruction using an in vitro ureter-stent model. BMC Urol. 2021;21(1):100. doi:10.1186/s12894-021-00865-w 


\section{Publish your work in this journal}

Research and Reports in Urology is an international, peer-reviewed, open access journal publishing original research, reports, editorials, reviews and commentaries on all aspects of adult and pediatric urology in the clinic and laboratory including the following topics: Pathology, pathophysiology of urological disease; Investigation and treatment of urological disease; Pharmacology of drugs used for the treatment of urological disease. The manuscript management system is completely online and includes a very quick and fair peer-review system, which is all easy to use. Visit http://www.dovepress.com/ testimonials.php to read real quotes from published authors. 